\title{
Interaction between Duration of Activity and Time Course of Recovery from Slow Inactivation in Mammalian Brain $\mathrm{Na}^{+}$Channels
}

\author{
Amir Toib, Vladimir Lyakhov, and Shimon Marom \\ The Bernard Katz Minerva Center for Cell Biophysics, Department of Physiology, Faculty of Medicine, Technion, and The \\ Rappaport Institute for Research in the Medical Sciences, Haifa 31096, Israel
}

Nall and NallA channels are the most abundant voltage-gated channels in neonatal and adult cortex, respectively. The relationships between activity and availability for activation of these channels were examined using the Xenopus expression system. The main point of this work is that the time constant $(\tau)$ of recovery from the unavailable (inactivated) pool is related to the duration $(t)$ of previous activation by a power law: $\tau(t)=p \cdot t^{D}$, with a scaling power $D$ congruent to 0.8 and 0.5 for Nall and NallA, respectively, and $p$ as a constant kinetic setpoint. These relationships extend from tens of milliseconds to several minutes and are intrinsic to the channel protein. Coexpression of $\beta 1$ auxiliary subunit, together with the $\alpha$ subunit of the NallA channel, modulates the constant kinetic setpoint but not the scaling power of the latter. The power law scaling between activity and availability is not a universal property of ion channels; unlike that of voltage-gated sodium channels, the rate of recovery from slow inactivation of the ShakerB channel is virtually insensitive to the duration of previous stimuli. It is suggested that the power law scaling described here can act as a molecular memory mechanism that preserves traces of previous activity, over a wide range of time scales, in the form of modulated reaction rates. This mechanism should be considered when theorizing about the dynamics of threshold and firing patterns of neurons.

Key words: sodium channel; ion channel; inactivation; excitability; power law; scaling
Modulation of threshold potential by changes in the availability of conductances is a powerful memory mechanism that depends solely on the intrinsic properties of a neuron, independent of changes in synaptic weight. In the past 30 years, there have been many reports of slow changes in the availability of voltage-gated sodium channels by means of a slow inactivation process (e.g., Adelman and Palti, 1969; Chandler and Meves, 1970; Fox, 1976; Schauf et al., 1976; Brismar, 1977; Rudy, 1978; Almers et al., 1983; Simoncini and Stühmer, 1987; Stühmer et al., 1987; Ruben et al., 1992; Cummins and Sigworth, 1996; Featherstone et al., 1996; Fleidervish et al., 1996; Hayward et al., 1997). Slow inactivation of voltage-gated sodium channels was found in all membranes in which appropriate experiments have been conducted (Ruben et al., 1992) and extends over a wide range of time scales (tens of milliseconds to minutes). The wide range of reported time scales of slow inactivation, together with the fact that the availability of voltage-gated sodium channels is a key determinant of threshold potential, prompted us to examine the relationships between activity and availability for activation of these channels. More specifically, we explored the possibility that a scaling relationship [Bassingthwaighte et al. (1994), their references] exists between the reaction rates involved in slow inactivation and the duration of previous depolarizations. A scaling relationship, in this context, means that there are no characteristic reaction rates for inactivation; instead, the effective rate constants reflect the time allowed for inactivation to occur. Voltage-gated sodium

Received Nov. 3, 1997; revised Dec. 11, 1997; accepted Dec. 15, 1997.

This work was partially supported by a grant from the Israel Science Foundation. We thank E. Braun, D. Dagan, Y. Palti, and I. Perlman for their help throughout the preparation of this manuscript.

Correspondence should be addressed to Dr. Shimon Marom, Department of Physiology, Faculty of Medicine, Technion, Efron Street, Bat-Galim, P.O. Box 9697 Haifa 31096, Israel.

Copyright (C) 1998 Society for Neuroscience $\quad 0270-6474 / 98 / 181893-11 \$ 05.00 / 0$ channels are usually regarded as either (1) not important for long-term modulations extending beyond the envelope of an action potential or (2) conferring a uniquely defined time scale that can be directly derived from the process of slow inactivation. However, if the rates of slow inactivation of voltage-gated sodium channels are linked to the history of activity via a scaling relationship, the role of these channels in activity-dependent processes must be reexamined, because they can participate in forms of cellular memory over all available time scales.

Types II and IIA voltage-gated sodium channels (Noda et al., 1986; Auld et al., 1988) are the most abundant voltage-gated sodium channels in the mammalian brain (Catterall, 1992). This report shows that the time constant $(\tau)$ for recovery of these channels from slow inactivation is intrinsically related to the duration $(t)$ of previous history of depolarizations by a power law: $\tau(t)=p \cdot t^{D}$, with a constant kinetic setpoint $(p)$ and a positive scaling power $(D)$ over a wide range of time scales.

\section{MATERIALS AND METHODS}

\section{Expression system}

Currents were measured from the membranes of Xenopus oocytes injected with cRNA coding for the brain channel types NaII (Noda et al., 1986) or NaIIA (Auld et al., 1988) or for the rapidly inactivating ShakerB potassium channel (Tempel et al., 1987). cRNA for the rat brain $\beta 1$ subunit [Isom et al. (1994), their references] was kindly given by I. Lotan (Wallner et al., 1993). Methods of DNA and cRNA preparation are standard. Oocytes were harvested from mature Xenopus females that were anesthetized by bathing in ice-cold water. After the surgery, frogs were allowed to recover for several hours in a small pool of water, and when fully active, they were returned to the main tank. Oocytes were dissociated in $2 \mathrm{mg} / \mathrm{ml}$ collagenase 1A (Sigma, St. Louis, MO). Stage $\mathrm{V}-\mathrm{VI}$ defolliculated cells were injected with $0.4-40 \mathrm{nl}$ of mRNA $(\approx 1$ $\mathrm{mg} / \mathrm{ml}$ ). In coexpression experiments, the $\beta 1 \mathrm{cRNA}$ was coinjected with the $\alpha$ subunit at a molar ratio of $\cong 1: 1$. Injected cells were incubated at $18^{\circ} \mathrm{C}$ for up to $7 \mathrm{~d}$ in frog Ringer's solution. 
$A$
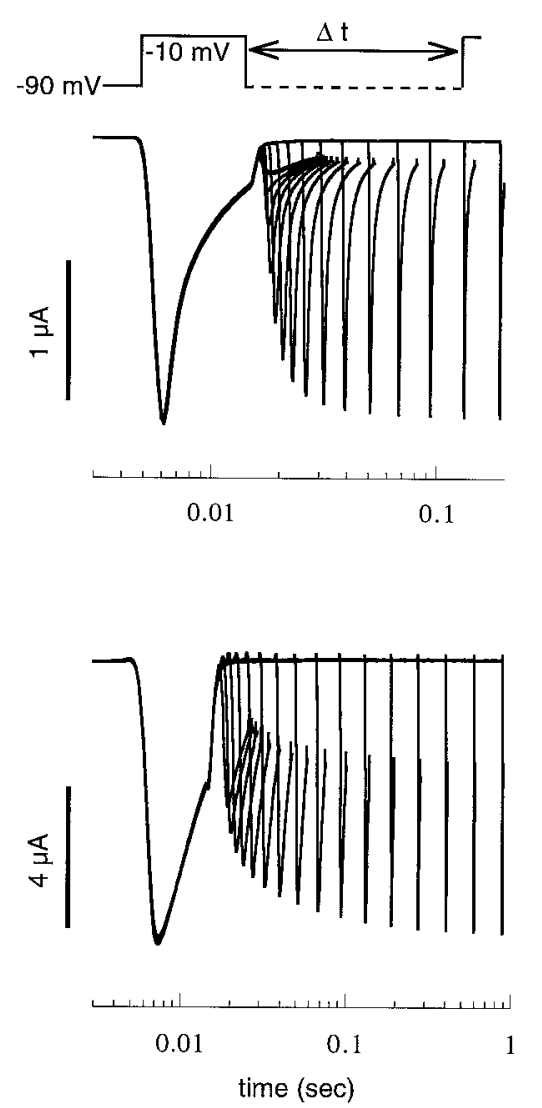

$B$
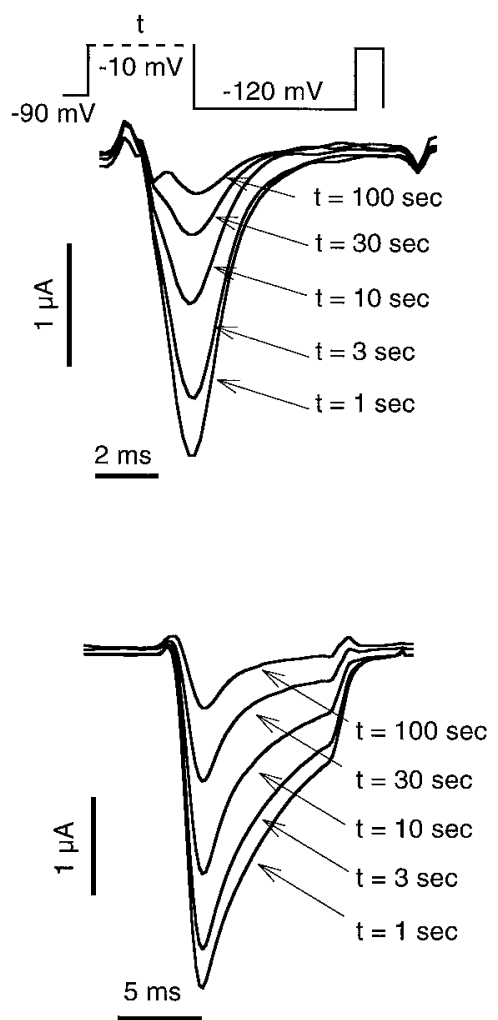

C

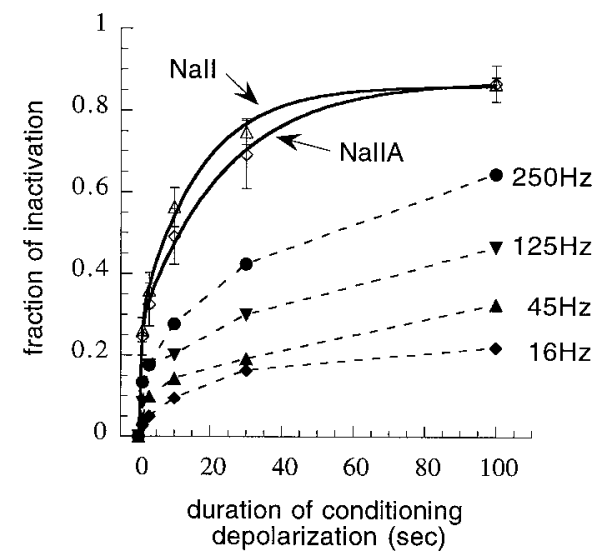

Figure 1. Inactivation of voltage-gated sodium channels. $A$, Time scales of rapid inactivation rates, for both NaII (top) and NaIIA (bottom) channels, are demonstrated using a double-pulse protocol. The membrane is pulsed to $-10 \mathrm{mV}$ from a holding potential of $-90 \mathrm{mV}$ (pulse duration is $10 \mathrm{msec}$ ). The membrane potential of the interval between the first and the second pulses is $-90 \mathrm{mV}$, and its duration is logarithmically increased (note the time axes). The time constants of recovery from rapid inactivation at -90 and $-120 \mathrm{mV}$ are summarized in Table 1 . B, Representative families of current traces showing the level of slow inactivation are presented. Oocytes are pulsed from a holding potential of -90 to $-10 \mathrm{mV}$ for a duration $t$. After this conditioning depolarization, the oocyte membrane potential is stepped to $-120 \mathrm{mV}$, allowing for recovery from the rapid inactivation process (6 or 100 msec for NaII and NaIIA, respectively; see Materials and Methods, Table 1). The availability of the channels for activation is then tested by an application of a short depolarizing test pulse to $-10 \mathrm{mV}$. The resulting test pulse current traces are shown for both channel subtypes (top, NaII; bottom, NaIIA); note the different time-scale bars (top and bottom). The durations ( $t$ values) of the conditioning pulses are depicted accordingly. $C$, Kinetics of entry into the slow inactivation state is shown for NaII (open triangles; $n=6 ; \pm \mathrm{SD}$ ) and NaIIA (open diamonds; $n=5$; \pm SD), together with double exponential functions depicted by continuous lines $\left(\mathrm{NaII}, \tau_{1}=0.6\right.$; fraction $=0.33 ; \tau_{2}=16.3$; fraction $=0.67 ; R>0.99 ;$ NaIIA, $\tau_{1}=0.6$; fraction $=0.31 ; \tau_{2}=21.9$; fraction $=0.69 ; R>0.99$ ). The time course of slow inactivation of NaIIA channels, in response to physiologically realistic stimulation patterns, is depicted by broken lines. In these experiments, during the conditioning phase, the membrane is held at $-60 \mathrm{mV}$ and pulsed to $0 \mathrm{mV}$ for $2 \mathrm{msec}$ at various frequencies ( filled diamonds, $16 \mathrm{~Hz}$; filled triangles, $45 \mathrm{~Hz}$; filled inverted triangles, $125 \mathrm{~Hz}$; filled circles, $250 \mathrm{~Hz}$ ). Note that although the main effect of a pulse train is completed within the first $30 \mathrm{sec}$ (i.e., 480 pulses at $16 \mathrm{~Hz}$ to 7500 pulses at $250 \mathrm{~Hz}$ ), accumulation of inactivation continues throughout the examined range (i.e., 1600 pulses at $16 \mathrm{~Hz}$ to 25000 pulses at $250 \mathrm{~Hz}$ ).

\section{Electrophysiological measurements}

The resulting conductances were studied with patch-clamp (Hamill et al., 1981) and two-electrode voltage-clamp (TEVC) techniques, using Axopatch 200A and GeneClamp 500 amplifiers (Axon Instruments, Foster City, CA), respectively. Pipets for patch-clamp experiments were made from glass (\#7052; Garner Glass Co., Claremont, CA) that was polished to 3-5 $\mathrm{M} \Omega$. Solutions for patch-clamp recordings were (in $\mathrm{mM}$ ): $96 \mathrm{NaCl}$, $2 \mathrm{KCl}, 1 \mathrm{CaCl}_{2}, 1 \mathrm{MgCl}_{2}$, and $10 \mathrm{HEPES}, \mathrm{pH} 7.5$, and $95 \mathrm{KCl}, 5 \mathrm{NaCl}$,

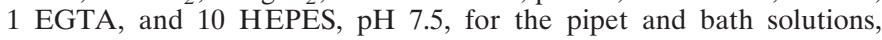
respectively. Intracellular aluminum silicate glass electrodes for TEVC experiments were filled with $2 \mathrm{M} \mathrm{KCl}$, resulting in resistances ranging from 0.5 to $2 \mathrm{M} \Omega$. The bath solution for TEVC experiments was (in $\mathrm{mM}$ ): $95 \mathrm{NaCl}, 3 \mathrm{KCl}, 2 \mathrm{CaCl}_{2}$, and $10 \mathrm{HEPES}, \mathrm{pH}$ 7.5. Experiments were handled by a Quadra 800 (Apple Computers, Inc.) with PULSE software (HEKA Electronic) and an ITC-16 computer interface (Instrutech Corporation). Data were low-pass filtered at $2-5 \mathrm{kHz}$ and sampled at $10-25$ $\mathrm{kHz}$. Long trains of pulses were delivered by a homemade pulse generator that was triggered from the PULSE software. P/n leak subtraction routines of the PULSE software were used. Some of the experiments involved very long voltage pulses that caused nonspecific conductances to appear. These conductances relaxed completely within $2 \mathrm{sec}$ at a hyperpolarized membrane potential. Continuous perfusion during very long conditioning pulses $(>3 \mathrm{sec})$ speeds up this relaxation.

\section{Pulse protocols and analysis of slow recovery in voltage-gated sodium channels}

(1) The time constants for recovery from rapid inactivation (Hodgkin and Huxley, 1952) were characterized at two voltages, -90 and -120 $\mathrm{mV}$, by a standard double-pulse protocol.

(2) The kinetics of entry into the slow inactivation state was examined. In these experiments, a conditioning pulse from -90 to $-10 \mathrm{mV}$ was applied to the membrane for various durations $(1,3,10,30$, and $100 \mathrm{sec})$. Each conditioning pulse was followed by a recovery interval at $-120 \mathrm{mV}$ for either 6 or $100 \mathrm{msec}$ for NaII and NaIIA, respectively. This interval, at $-120 \mathrm{mV}$, allowed for a practically complete recovery of the channels from rapid inactivation in the case of the NaII $\alpha$ subunit $[\mathrm{NaII}(\alpha)]$ and the brain $\beta 1$ subunit of the Na channel coexpressed with the NaIIA $\alpha$ subunit $[\operatorname{NaIIA}(\alpha+\beta 1)]$ and for $\cong 90 \%$ recovery in the case of the 


\begin{tabular}{|c|c|c|c|c|c|}
\hline & $\mathrm{V}(\mathrm{mV})$ & $\tau_{1}(\mathrm{msec})$ & $A_{1}$ & $\tau_{2}(\mathrm{msec})$ & $A_{2}$ \\
\hline \multirow[t]{2}{*}{$\mathrm{NaII}(\alpha)$} & -90 & $\begin{array}{l}3.12 \pm 0.02 \\
(n=3)\end{array}$ & & & \\
\hline & -120 & $\begin{array}{l}1.25 \pm 0.03 \\
(n=3)\end{array}$ & & & \\
\hline \multirow[t]{2}{*}{ NaIIA $(\alpha)$} & -90 & $\begin{array}{l}4.14 \pm 1.95 \\
(n=9)\end{array}$ & $0.46 \pm 0.18$ & $\begin{array}{l}113.5 \pm 81.6 \\
(n=9)\end{array}$ & $0.54 \pm 0.02$ \\
\hline & -120 & $\begin{array}{l}7.79 \pm 2.39 \\
(n=6)\end{array}$ & $0.40 \pm 0.03$ & $\begin{array}{l}67.5 \pm 21.9 \\
(n=6)\end{array}$ & $0.60 \pm 0.02$ \\
\hline \multirow[t]{2}{*}{ NaIIA $(\alpha+\beta 1)$} & -90 & $\begin{array}{l}4.98 \pm 0.50 \\
(n=4)\end{array}$ & & & \\
\hline & -120 & $\begin{array}{l}1.30 \pm 0.06 \\
(n=3)\end{array}$ & & & \\
\hline
\end{tabular}

Rates of recovery from the rapid inactivation states are derived by fitting an exponential function to the maxima of inward currents during the recovery phase in double-pulse experiments (as demonstrated in Fig. $1 A$ ). In the case of NaIIA $(\alpha$ subunit only) channel, fitting necessitates at least two exponential terms. One exponential term is sufficient in the case of NaII and in the case of NaIIA isoform that is coexpressed with a $\beta 1$ subunit. Time constants $(\tau)$, relative amplitudes $(A)$, SDs, and number of experiments are given for -90 and $-120 \mathrm{mV}$.

NaIIA $\alpha$ subunit $[\operatorname{NaIIA}(\alpha)]$. A test pulse to $-10 \mathrm{mV}$ was applied immediately after the recovery interval. The fraction of channels in the slow inactivated state was calculated from the ratio between the measured peak current response to the test pulse and the peak current response evoked by a similar procedure after a $15 \mathrm{msec}$ conditioning pulse.

(3) Two types of pulsing protocols aimed at exploring the kinetics of recovery from slow inactivation were performed.

Recovery from very long conditioning pulses. In these experiments, a conditioning pulse from -90 to $-10 \mathrm{mV}$ was applied to the membrane for various durations $(3,10,30,100$, and $300 \mathrm{sec})$. Each conditioning pulse was followed by a $1 \mathrm{sec}$ recovery interval at $-90 \mathrm{mV}$. This interval allowed for a practically complete recovery of the channels from rapid inactivation and was followed by a series of short test pulses aimed at monitoring the process of recovery from slow inactivation. The test pulses from -90 to $-10 \mathrm{mV}$ were delivered at a frequency of $0.33,0.5$, or $1 \mathrm{~Hz}$; no effect of the frequency of the test pulses on the kinetics of recovery from slow inactivation was observed within this range (which is an order of magnitude lower than the slowest observed rate of recovery from rapid inactivation).

Recovery from shorter conditioning pulses. In these experiments, the duration of the conditioning pulse $(10,30,100,300,1000$, and $3000 \mathrm{msec})$ allowed a standard double-pulse protocol to be used to follow the time course of recovery. Each pair of pulses was composed of a conditioning pulse from -90 to $-10 \mathrm{mV}$ followed by a logarithmically varying recovery period at $-90 \mathrm{mV}$ and a test pulse to $-10 \mathrm{mV}$. Note the overlap (at a conditioning duration of $3 \mathrm{sec}$ ) between the two types of pulsing protocols.

The peak current amplitude of each $n$th test pulse $\left(I_{n}\right)$ was observed on-line, and the pulsing was stopped by the experimenter when $I_{n}$ approached a maximum value $\left(I_{\max }\right)$. The fraction of inactivation $F$ at the time of the $n$th test pulse was normalized to: $F=1-\left(I_{n}-I_{1}\right) /\left(I_{\max }\right.$ $\left.-I_{1}\right)$, where $I_{1}$ is the peak amplitude of the current that was evoked by the first test pulse. The time constant $\tau$ was extracted by fitting a sum of exponential functions to $F$. Fitting was done using KaleidaGraph (Synergy Software).

\section{RESULTS}

Time-scale separation between rapid (HodgkinHuxley-like) and slow inactivation processes

Voltage-gated sodium channels inactivate rapidly in response to a short, fully activating depolarizing voltage pulse. This phenomenon, which has been described and analyzed in the literature since the publication of the Hodgkin and Huxley study (1952), occurs at the milliseconds time scale and is demonstrated in Figure $1 A$ and summarized in Table 1 . The subject of the present report is a slower inactivation process, which involves reaction rates at the seconds-to-minute time scale and is demonstrated in Figure $1 B$. In these experiments, the membrane is pulsed to a fully activating membrane potential for a duration $t$, followed by a hyperpolarizing segment that is long enough to allow recovery from the rapid Hodgkin-Huxley inactivation process. The latter segment is then followed by a short depolarizing test pulse. The fraction of inactivated channels, as a function of $t$, is measured from the peak amplitude of the current that was evoked by the test pulse. As indicated by the decay of availability in the experiments of Figure $1 C$, within the examined range of up to 100 -seclong depolarization pulses, the channels are shifted into an unavailable pool following uniquely defined reaction rates at the seconds time scale (double exponential fits are depicted as continuous lines).

Because the membrane potential of a neuron under physiological conditions is not expected to be depolarized for seconds and because this study is aimed at making a statement about the physiological significance of slow inactivation, NaIIA channels were exposed to stimulation protocols that are similar in amplitude, duration, and frequencies to firing patterns in neocortical neurons. The results (Fig. $1 C$, dashed lines) show that when the membrane is held at $-60 \mathrm{mV}$ and pulsed to $0 \mathrm{mV}$ for $2 \mathrm{msec}$ at frequencies ranging from 16 to $250 \mathrm{~Hz}$, the reduction of the availability of the channels for activation is significant. It is important to note that the pulse train data are fundamentally different from the continuous data; the pulse train protocol, especially at high frequencies, induces accumulation of channels in both rapid as well as slow inactivation states and therefore cannot directly point at the physiological significance of slow inactivation alone. The low-frequency data of Figure $1 C$ is more informative in that context. In the experiments that are described in the rest of this report, the pulses to induce slow inactivation (defined here as conditioning pulses) are continuous and optimized for kinetic characterization rather than for physiological compatibility.

\section{The time course of recovery from slow inactivation is scaled according to the duration of the conditioning pulse}

The time-scale separation, between the milliseconds range of rapid activation-inactivation (Fig. $1 A$, Table 1 ) and the many- 
$A$

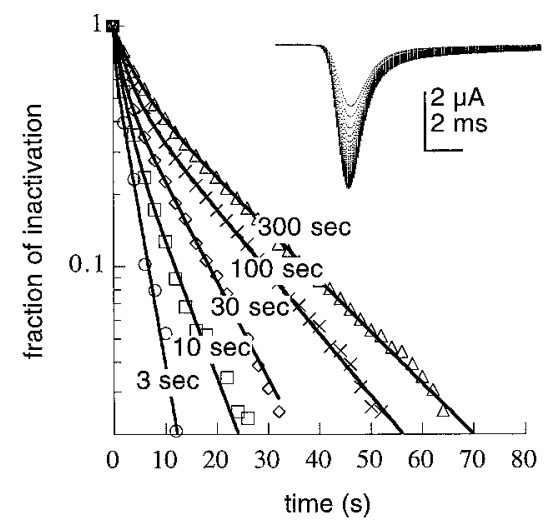

$B$

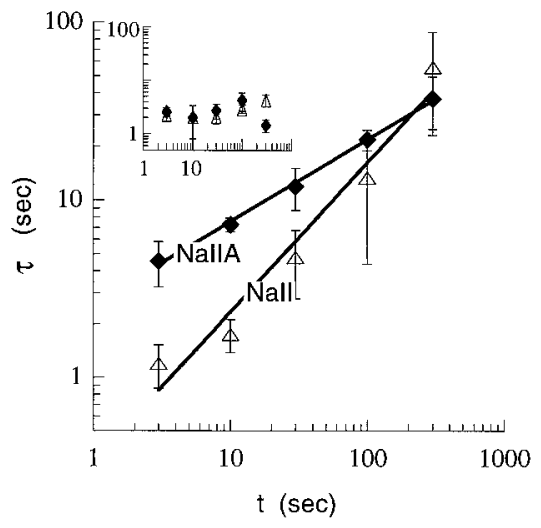

$D$
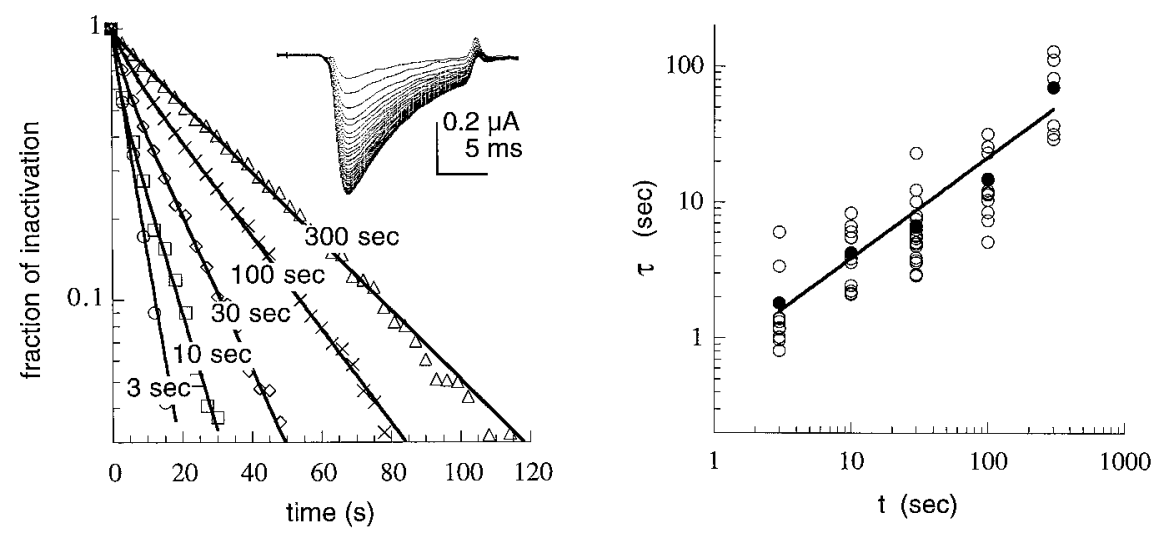

$C$

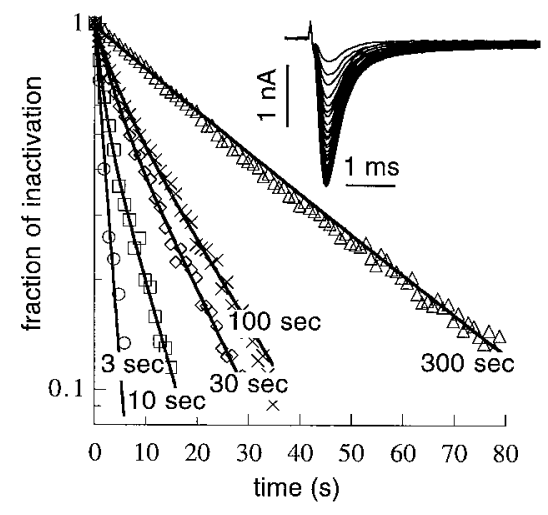

Figure 2. Scaling relationship between the duration of activation and the recovery rate from slow inactivation in voltage-gated sodium channels. $A$, Time course of recovery from slow inactivation induced by 3-, 10-, 30-, 100-, and 300-sec-long $(t)$ conditioning depolarizations (top, NaII; bottom, NaIIA). The recovery interval, at $-90 \mathrm{mV}$, from the end of the conditioning pulse to the beginning of the series of test pulses lasted 1 sec. The fraction of inactivation was normalized and plotted as described in Materials and Methods. Solid lines are double exponential fits to the data. Insets, Recovery from a 100-sec-long conditioning depolarization from -90 to $-10 \mathrm{mV}$. Recovery from slow inactivation is seen as a gradual increase in peak current responses to test pulses of $15 \mathrm{msec}$ duration from -90 to $-10 \mathrm{mV}$, delivered at a frequency of $0.5 \mathrm{~Hz}$ (top) or $0.33 \mathrm{~Hz}$ (bottom). B, The value of the slower time constant for recovery from slow inactivation $(\tau)$ shown increasing systematically as a function of the duration $t$ of the conditioning depolarization in NaII $(n>6$; $\pm \mathrm{SD})$ and NaIIA $(n=5 ; \pm \mathrm{SD})$ channel subtypes. Time constants were extracted from the time course of recovery as shown in $A$, with a test pulse frequency of $0.5 \mathrm{~Hz}$. Solid lines are power law functions of the form $\tau(t)=p \cdot t^{D}$ that were fit to the data (NaII, $p=0.3 ; D=0.84 ; R=0.99$; NaIIA, $p=2.6 ; D=0.46 ; R>0.99)$. Inset, The value of the shorter time constant as a function of conditioning depolarization in NaII (open triangles; $n>5$; $\pm \mathrm{SD}$ ) and NaIIA ( filled diamonds; $n>3 ; \pm \mathrm{SD}$ ) channel subtypes. The relative amplitudes of this recovery component (in the absence of the $\beta 1$ subunit) are within the range of $0.29-0.53$ and $0.09-0.33$ for NaII and NaIIA, respectively, and do not change systematically as the conditioning-pulse duration is increased. $C$, Time course of recovery from slow inactivation of NaII channels recorded in detached-macropatch configuration (i.e., the channels are not in contact with intracellular factors). Inactivation was induced by 3-, 10-, 30-, 100-, and 300-sec-long conditioning depolarizations. The recovery interval, at $-90 \mathrm{mV}$, from the end of the conditioning pulse to the beginning of the series of test pulses lasted $1 \mathrm{sec}$. Solid lines are double exponential fits to the data. Inset, Recovery from a $30 \mathrm{sec}$ conditioning depolarization from -90 to $-10 \mathrm{mV}$. Recovery from slow inactivation is seen as a gradual increase in peak current responses to test pulses of $10 \mathrm{msec}$ duration from -90 to $-10 \mathrm{mV}$, delivered at a frequency of $1 \mathrm{~Hz}$. $D$, The value of the slower time constant $(\tau)$ increasing systematically as a function of $t$. Mean values ( filled circles) are fitted with a power law of the form: $\tau(t)=p \cdot t^{D}(p=0.7$; $D=0.75 ; R=0.97)$.

seconds range of slow inactivation (Fig. $1 B, C$ ), allows one to reduce the kinetic scheme of the system to a simplified two-states scheme, $A \Leftrightarrow I$, where $A$ stands for the set of all available-for-ionconduction states, and $I$ stands for all unavailable (inactivated) states. In this simplified picture, the classical Hodgkin-Huxley rapid inactivation ( $h$ gate) belongs to the available set of states. The rate of recovery $I \Rightarrow A$ is studied by applying a series of short depolarizing test pulses after $t$ long conditioning depolarizations. In these experiments, all the intervals between depolarizations are at the seconds time scale; this is essential to diminish the interference of fast gating processes, within the internal structure of $A$, with the process of recovery from slow inactivation. The results are shown in Figure 2. The time course of recovery from slow inactivation, induced by 3-, 10-, 30-, 100-, and 300-sec-long conditioning depolarizations, is extracted from the change in the peak of the current amplitude evoked by short depolarizing test pulses as explained in Materials and Methods. The recovery can be adequately described by a double exponential function $(R>$ 0.99) (Fig. 2A). The relationship between the two components of recovery from inactivation will be explored in a separate section of the Results, in which experimental protocols that are optimized for that aim are analyzed. Regardless of this relationship between the components, it is clear from Figure $2 A$ that the time course of recovery from inactivation is sensitive to the conditioning-pulse duration. In particular, the value of the slower time constant $(\tau)$ for $I \Rightarrow A$ relaxation increases systematically as a 
$A$

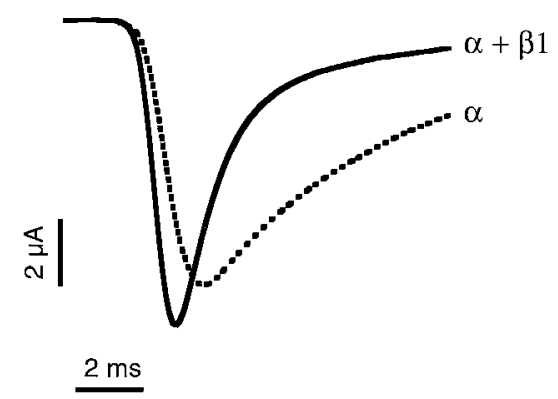

$B$

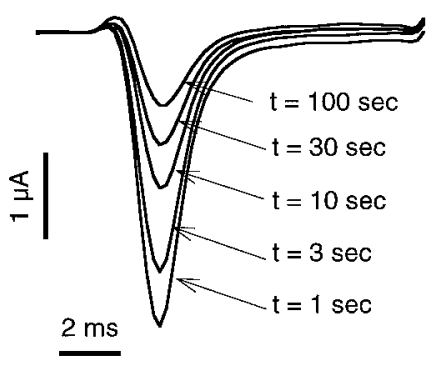

$C$

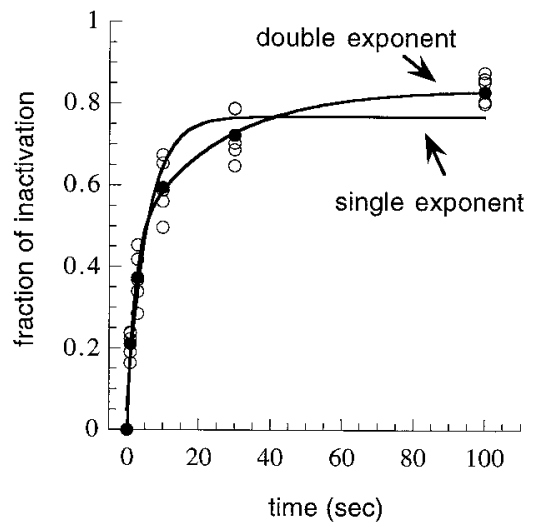

Figure 3. Effect of the $\beta 1$ subunit on the entry of NaIIA to slow inactivation. $A$, Typical responses of NaIIA $(\alpha)$ and NaIIA( $\alpha+\beta 1)$ channels to a depolarization pulse from -90 to $-10 \mathrm{mV}$. These traces were recorded from different oocytes (of the same batch) $3 \mathrm{~d}$ after injection with cRNA of the $\alpha$ subunit alone (dashed line) or together with cRNA of the $\beta 1$ subunit (continuous line) in a $\cong 1: 1$ molar ratio. The presence of $\beta 1$ subunit results in a faster activation and inactivation kinetics. No systematic effect on current amplitude was observed. $B$, A representative family of current responses to test pulses from -120 to $-10 \mathrm{mV}$, delivered $100 \mathrm{msec}$ after the end of a $t$ long conditioning depolarization to $-10 \mathrm{mV}$, showing the level of slow inactivation of $\operatorname{NaIIA}(\alpha+\beta 1)$ channels. $C$, Kinetics of entry into the slow inactivation state for NaIIA $(\alpha+\beta 1)$ channels (open symbols). For comparison, two types of exponential functions are fitted to the mean values ( filled symbols), a single exponent $(\tau=4.8 \mathrm{sec} ; R=0.92)$ and a double exponent $\left(\tau_{1}=2.12\right.$; fraction $=0.55 ; \tau_{2}=24.3$; fraction $\left.=0.45 ; R>0.99\right)$.

function of the duration $(t)$ of the conditioning pulse. A collection of many values of $\tau$ versus $t$ is shown on a log-log plot in Figure $2 B$, indicating that the longer the conditioning depolarization is, the slower the time constant for recovery from inactivation becomes. The behavior of the time constant as a function of conditioning-pulse duration is the key observation of the present report.

The mean values of the slower time constants $(\tau)$ for recovery from slow inactivation are related to $t$ by a power law (Fig. $2 B$, solid lines) of the form:

$$
\tau(t)=p \cdot t^{D},
$$

suggesting the existence of a scaling relationship between the recovery rate from the unavailable set of states and the duration of previous activity.

\section{Scaling of recovery time course is intrinsic to the $\alpha$ subunit of the channel protein}

Do the above-described relationships between activity and availability arise from an intrinsic (physicochemical) channel machinery? The power law scaling could stem from the Xenopus oocyte intracellular milieu via a biochemical path (e.g., enzymatic modulation that is activated by long depolarizing pulses) that might be entirely irrelevant to the neurophysiological case. Taking advantage of the fact that NaII channels are efficiently expressed in oocytes, allowing one to record macroscopic $\mathrm{Na}$ currents in a detached patch of membrane, we show in the experiments of Figure 2, $C$ and $D$, that the power law relationships between $\tau$ and $t$ are not produced by changes in the intracellular environment of the oocyte during the conditioning phase; the scaling phenomenon is clearly seen in the detached-patch mode, in which the channel protein is not in contact with the cellular environment. There are some differences between the results of TEVC and patch-clamp recordings, especially at the faster time scales (compare Fig. $2 C$ with $A$, top). These differences might be related to the compromised accuracy of the TEVC technique in describing the fast sodium channel kinetics [Ruben et al. (1997), their references]. However, it is important to note that these differ- ences do not interfere with the key observation of the present report (compare Fig. $2 B$ with $D$ ).

\section{Coexpression of the $\alpha$ subunit together with the $\beta 1$ subunit introduces a kinetic offset in the relationship between the duration of activity and the time course of recovery}

The Na channel isoforms studied here have a much slower inactivation kinetics, compared with their kinetics in mammalian cells, when expressed in Xenopus oocytes in the absence of a $\beta$ subunit [Isom et al. (1994), their references]. This becomes a major concern if one is trying to generalize results on $\mathrm{Na}$ channel inactivation from Xenopus oocyte experiments to neurons. To overcome this problem, we conducted a set of experiments in which the rat brain $\beta 1$ subunit of the Na channel was coexpressed with the NaIIA $\alpha$ subunit. The modulatory effect of the $\beta 1$ subunit on the fast kinetics of NaIIA is demonstrated in Figure $3 A$. Slow inactivation of $\operatorname{NaIIA}(\alpha+\beta 1)$ channels is demonstrated in Figure $3 B$. Current responses to a short depolarizing test pulse decrease in amplitude as the duration of a preceding $t$-sec-long depolarization conditioning pulse is increased. Figure $3 C$ summarizes the kinetics of entry of $\operatorname{NaIIA}(\alpha+\beta 1)$ channels into the slow inactivation state. The onset of slow inactivation of NaIIA channels, in the presence of the $\beta 1$ subunit, consists of two components. These two components have comparable amplitudes but distinctly separated time scales. An attempt to describe the entry kinetics by a single exponent yields a less agreeable approximation (depicted in Fig. 3C). The data of Figure 3 suggest that in contrast to the fast inactivation kinetics, relaxation of the NaIIA into slow inactivation is not markedly altered by $\beta 1$ (compare Figs. $3 C$ with $1 C$ ). As shown in Figure 4, the dependence of the slower rate of recovery of the $\operatorname{NaIIA}(\alpha+\beta 1)$ channel from slow inactivation on the duration of the conditioning pulse is also quite similar to that of the NaIIA channel. Interestingly, the kinetic setpoint $(p)$ is slightly smaller, 1.88 compared with 2.6 for $\operatorname{NaIIA}(\alpha+\beta 1)$ and $\operatorname{NaIIA}(\alpha)$, respectively. This small difference is statistically significant $(P=0.028$, paired two-sample $t$ test for means) and shows that $\operatorname{NaIIA}(\alpha)$ is slower to recover from inac- 
$A$

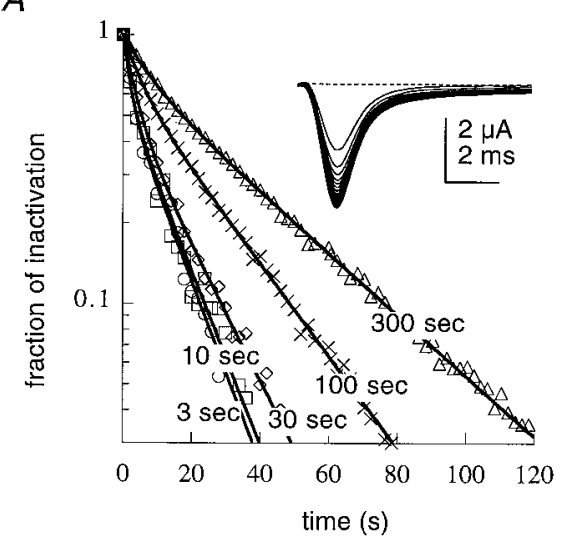

$B$

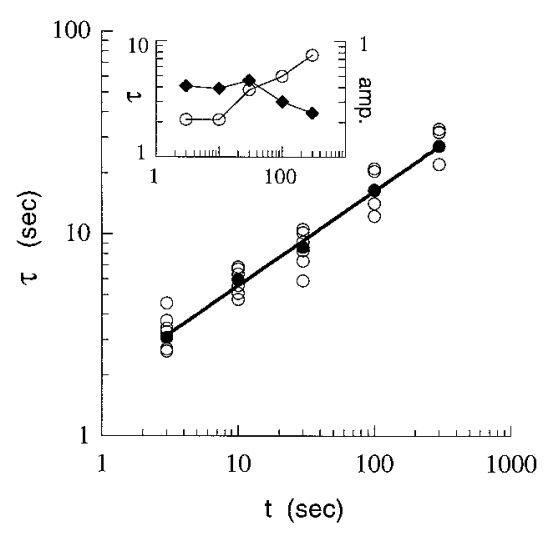

Figure 4. Scaling relationship between the duration of activation and recovery rate from slow inactivation in NaIIA channels that are coexpressed with the $\beta 1$ subunit. $A$, Time course of recovery from slow inactivation in $\operatorname{NaIIA}(\alpha+\beta 1)$ channels induced by 3-, 10-, 30-, 100-, and 300 -sec-long $(t)$ conditioning depolarizations. The recovery interval, at $-90 \mathrm{mV}$, from the end of the conditioning pulse to the beginning of the series of test pulses lasted $1 \mathrm{sec}$. The fraction of inactivation was normalized and plotted as described in Materials and Methods. Solid lines are double exponential fits to the data. Inset, Recovery from a $100 \mathrm{sec}$ conditioning depolarization. Recovery from slow inactivation is seen as a gradual increase in peak current responses to test pulses from -90 to -10 $\mathrm{mV}$, delivered at a frequency of $0.5 \mathrm{~Hz}$. $B$, The value of the slower time constant $(\tau)$ increasing systematically as a function of $t$. Time constants were extracted from the time course of recovery as shown in $A$, with a test pulse frequency of $0.5 \mathrm{~Hz}$. Filled circles are the mean values at each $t$. The solid line is a power law function of the form $\tau(t)=p \cdot t^{D}$ that was fit to the mean values ( $p=1.88 ; D=0.45 ; R>0.99)$. Inset, The relative amplitude ( filled diamonds, right $y$-axis) and time constant (open circles, left $y$-axis) of the faster component of recovery from slow inactivation, for the cell shown in $A$, as a function of conditioning-pulse duration.

tivation, relative to $\operatorname{NaIIA}(\alpha+\beta 1)$, over the entire range of conditioning durations tested here. Coexpression of the $\alpha$ subunit of the NaIIA channel together with the $\beta 1$ subunit uncovered another interesting feature that is apparently absent when the $\alpha$ subunit of the NaIIA channel is expressed alone; a close examination of the curves of Figure $4 A$ suggests that as the conditioning duration increases, the time constant of the faster recovery component seems to increase, whereas its relative amplitude decreases. This trend is demonstrated in the inset of Figure $4 B$ that shows the faster time constant and its amplitude as a function of conditioning duration for the case of Figure $4 A$. Note that in the absence of the $\beta 1$ subunit, there is no systematic relationship between the faster time constant for recovery from slow inactivation and the conditioning duration (Fig. $2 B$, inset). The interplay between the time constants and relative amplitudes of the different components of recovery from inactivation will be more fully addressed in other experiments (see Fig. 7).

\section{The scaling power is not affected by the pulse protocol used for recovery measurement}

In this study, the method for observing the time course of recovery is based on a series of brief depolarizing pulses at low frequencies (see Materials and Methods). This method is commonly used when the recovery time scales are very long (e.g., Cummins and Sigworth, 1996). Nevertheless, it is important to ascertain that recovery data are not distorted by the series of test pulses. A comparison was made to recovery data in which conditioning pulses were followed by a varying recovery interval and a single test pulse. A single set of 3-300-sec-long conditioning pulses, each of which was followed by a single recovery interval and a single test pulse, lasts $1.5-2$ hr. Figure 5 shows an exemplar $(5 A)$ and a summary $(5 B)$ of results from three oocytes that were injected with $\operatorname{NaIIA}(\alpha+\beta 1)$ channels. Recordings from these oocytes were stable throughout the entire pulse protocol. As shown in Figure 5, the scaling power between conditioning duration and recovery time constant is similar to the scaling power extracted from series of recovery pulses.

\section{The scaling relationship between conditioning duration and recovery time course persists over a range of voltages}

The scaling of recovery time course was shown above at only one voltage $(-90 \mathrm{mV})$. It is interesting to see whether the membrane potential, during the recovery phase, affects the scaling relationship between the duration of the conditioning pulse and the time course of recovery from inactivation. A comparison between recoveries at $-120,-90$, and $-60 \mathrm{mV}$ is presented in Figure 6 for the case of $\operatorname{NaIIA}(\alpha+\beta 1)$ channels. Evidently, the scaling relationship is not unique to a particular voltage; it persists over a wide range of voltages. As shown in Figure 6, changing the recovery voltage from -90 to $-120 \mathrm{mV}$ does not influence the dependence of the recovery time course on the duration of the conditioning depolarization. In contrast, when the membrane is held at $-60 \mathrm{mV}$ compared with the other two voltages during the recovery phase, the recovery time course becomes slower as the conditioning duration increases. This behavior at $-60 \mathrm{mV}$ might be related to the inability to separate (at this relatively depolarized level) between the different components of inactivation (see below).

\section{The relationships between different components of recovery from inactivation in voltage-gated sodium channels: rapid, intermediate, and slow kinetics}

The experiments described above show that recovery from inactivation in voltage-gated sodium channels is composed of at least three components. They are defined here as follows: a rapid component (Fig. $1 A$, Table 1), an intermediate component (which is observed as the first component of recovery in, e.g., Figs. $2 A$, $4 A$ ), and a slow component (which was at the focus of the present work). The time course of the slow component was shown above to be scaled according to the duration of the conditioning depolarization (Figs. $2 B, D, 4 B$ ). Up to this point, an effort was directed toward separating the slow component from the other, faster components to allow for an accurate analysis. Under natural conditions, however, these components overlap and operate 
$A$

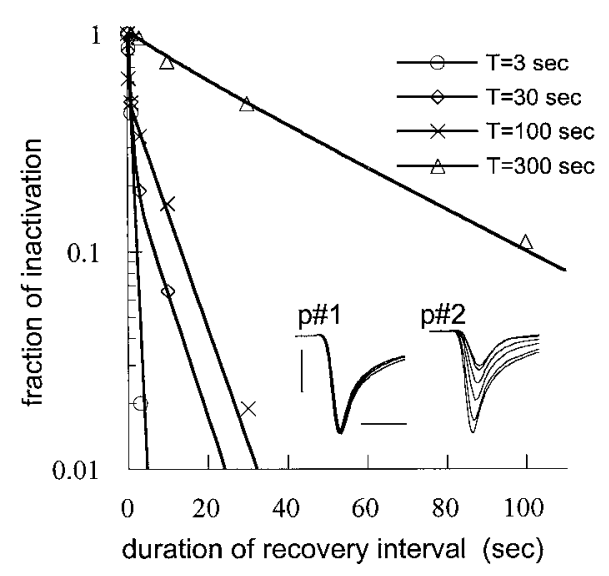

$B$

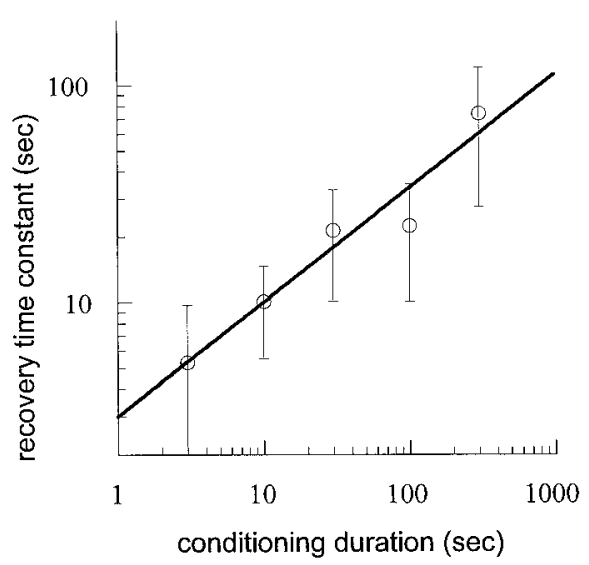

Figure 5. Scaling relationship between the conditioning duration and recovery time course in long double-pulse protocols with $\operatorname{NaIIA}(\alpha+\beta 1)$ channels. $A$, Exemplar recovery time courses that were extracted from double-pulse protocols as follows: A short test pulse was applied $1 \mathrm{sec}$ before each conditioning pulse. This short test pulse ( $p \# 1$ in the inset) served as a control level for the calculation of inactivation fraction (see Materials and Methods) and as a measure for full recovery between pairs of pulses. Each $T$-sec-long conditioning pulse was followed by a recovery interval at $-90 \mathrm{mV}$ and a single test pulse to $-10 \mathrm{mV}$ ( $p \# 2$ in the inset). This procedure was repeated for various recovery intervals and conditioning durations. Insets, Control and recovery pulse pairs for $300 \mathrm{sec}$ conditioning duration. Calibration: $4 \mathrm{msec}, 4 \mu \mathrm{A}$. B, Relationship between conditioning duration and recovery time constant fitted to a power law function of the form: $\tau(t)=p \cdot t^{D}(p=2.9 ; D=0.52 ; R=0.96)$. Means and SDs of recovery time constants are from three oocytes.

in concert to determine the time course of recovery from inactivation. The results of Figure 7 reveal part of the complexity of the relationships between the three recovery components in $\operatorname{NaIIA}(\alpha$ $+\beta 1)$ channels. Figure 7 shows the relative amplitudes and time constants of the rapid, intermediate, and slow recoveries as a function of conditioning duration $(t)$. This figure was generated by exposing oocytes to the complete battery of pulse protocols of recovery from inactivation (i.e., conditioning durations from 10 msec to $300 \mathrm{sec}$ ). The data were extracted using the two types of pulse protocols that are detailed in Materials and Methods; filled circles depict data from double-pulse experiments, whereas open circles depict data from pulse-series experiments. At the millisec- onds time scale, the rapid (Hodgkin-Huxley-like) inactivation is dominant (Fig. 7, top left). The time constant of this rapid component is virtually insensitive to the duration of the conditioning pulse (top right). As the conditioning-pulse duration increases to the several-seconds time scale, the intermediate component of recovery becomes dominant (Fig. 7, middle left). The time constant of this component is sensitive to the conditioning-pulse duration (middle right). At the tens-of-seconds time scale, the slow, time-dependent component takes over (Fig. 7, bottom). Note the hierarchic interplay between the relative amplitudes as the conditioning-pulse duration increases. A similar interplay between the relative amplitudes as a function of conditioning duration was recently reported by Hayward et al. (1997) for muscle voltage-gated sodium channels in a mammalian expression system. It is conceivable that if conditioning pulses longer than $300 \mathrm{sec}$ were applied, additional components would be uncovered. (Note that the difference between the measured amplitudes of the intermediate component in double-pulse and pulse-series methods at the $3 \mathrm{sec}$ conditioning duration is probably attributable to (1) the inclusion of the rapid component in the intermediate component while fitting pulse-series data and (2) the compromised accuracy of fitting our pulse-series data to a component that is shorter than $1 \mathrm{sec}$ ).

\section{The scaling relationship exhibited by voltage-gated sodium channels is not a universal property of ion channels: a comparison with the ShakerB channel}

Although this study is about the mammalian brain voltage-gated sodium channels, it is instructive to compare the results to the behavior of other channel types. Such a comparison with the classic ShakerB channel is described below, showing that the scaling relationship exhibited by voltage-gated sodium channels is not a universal property of ion channels. Like voltage-gated sodium channels, ShakerB potassium channels inactivate in a slow ("C-type") in addition to a longer (milliseconds, "N-type") process of inactivation (Hoshi et al., 1991). The inset to Figure $8 A$ shows a series of superposed current responses to identical depolarizing voltage pulses to $+40 \mathrm{mV}$, separated by $1.5 \mathrm{sec}$ intervals at $-90 \mathrm{mV}$. Note that $\mathrm{N}$-type inactivation recovers completely from one pulse to the next. (The symbols of Fig. $8 A$ depict different interpulse intervals.) As shown in Figure 8, $B$ and $C$, and unlike voltage-gated sodium channels, ShakerB channels demonstrate a uniquely defined time scale for recovery from slow (Ctype) inactivation. This time scale $(\cong 5 \mathrm{sec})$ is practically independent of the duration of previous depolarization.

\section{DISCUSSION}

\section{Physical interpretation}

This report shows that voltage-gated sodium channels recover from the unavailable inactivation pool at an effective rate that is related by a power law to the time spent in that pool. Power law scalings are characteristic of fractals. By itself, the fact that some aspects of the gating of the voltage-gated sodium channels can be described by a fractal is not a unique observation. Fractal behavior was described in the gating of several ion channels in the past, (Liebovitch and Sullivan, 1987) and there is an ongoing debate concerning the molecular mechanisms (and the corresponding structural correlates) that underlie this phenomenon [Bassingthwaighte et al. (1994), their references). On the one hand, some models were proposed in which the scaling relationship in ion channel gating is attributed to a non-Markovian process. On the other hand, considering the statistical and experimental uncer- 
$A$
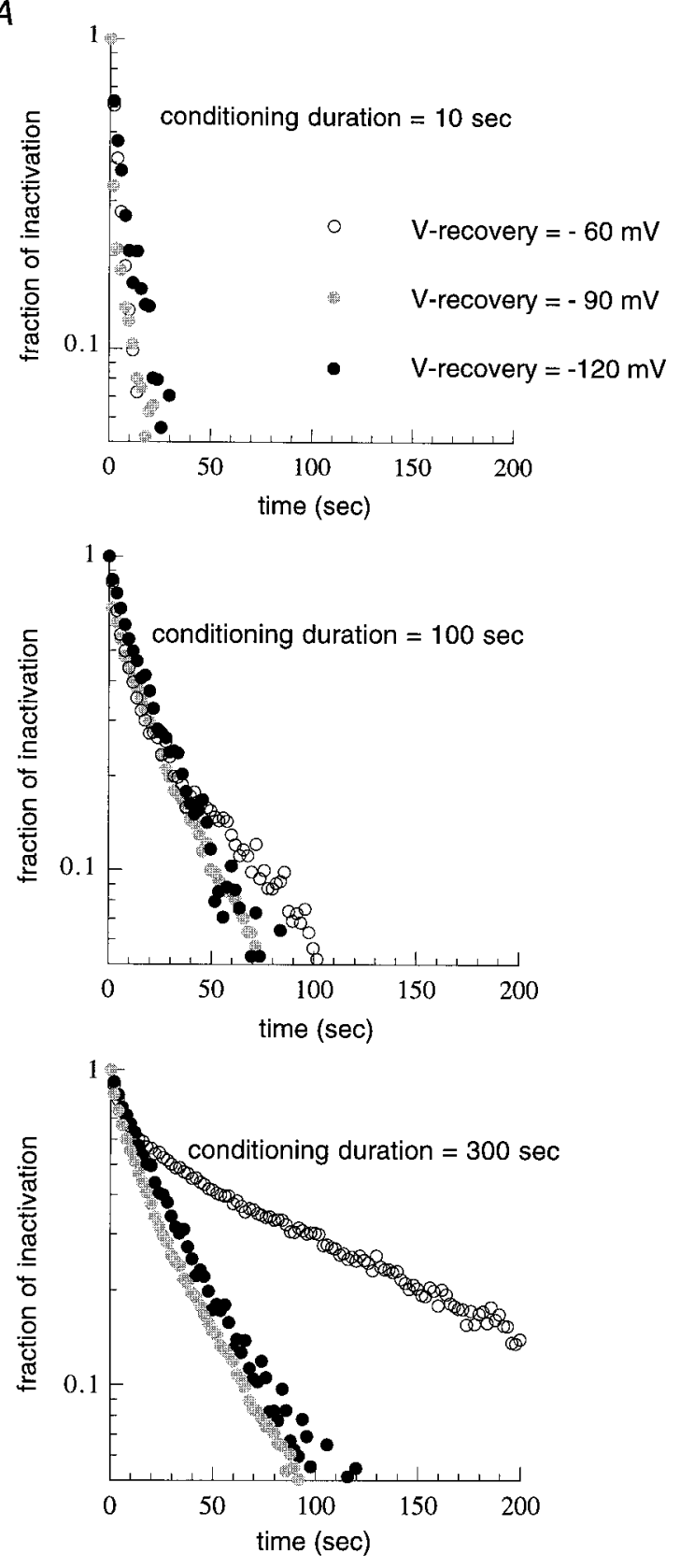

$B$

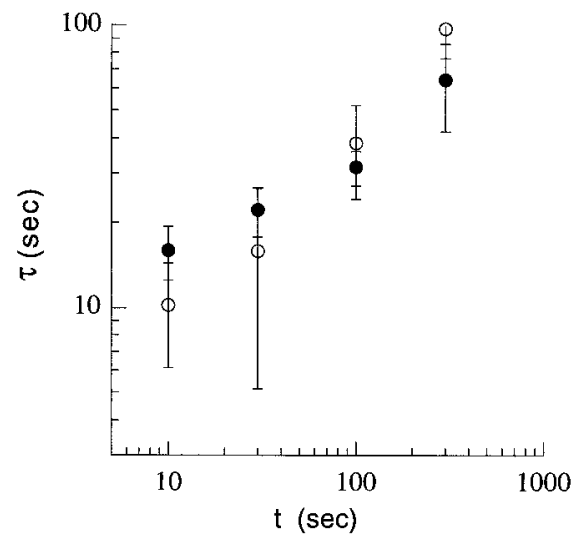

tainties that are associated with the extraction of time constants from long-lasting electrophysiological experiments, one should always keep in mind the possibility that power law scaling can arise from a (surprisingly small) sum of exponents (Sauve and Szabo, 1985). Unfortunately, there is no statistical way to compare the goodness of fit of these two theoretical models (Markovian and fractal) to the experimental data. As explained by Bassingthwaighte et al. (1994), the problem is that both of these models can have an arbitrary number of adjustable parameters; thus each model can be made to fit the experimental data to unlimited accuracy. In fact, this property of having an arbitrary number of adjustable parameters can be used to relate the two models to each other (Liebovitch, 1989). For example, one can think of the scaling relationship as resulting from a complex Markovian arrangement (with multiple time-independent rates) that is collapsed to (and described by) a single effective rate constant. Such an arrangement can have a branching form, a chain form, or any other complex form. Although it is not necessary to assume a particular kinetic scheme, the principle of collapsing a complex Markovian arrangement to a scaled effective rate can be exemplified by the following mechanism:

$$
A \stackrel{f(v)}{\longleftrightarrow} I_{1} \leftrightarrow I_{2} \leftrightarrow I_{3} \leftrightarrow I_{4} \leftrightarrow I_{5} \ldots \leftrightarrow I_{n} .
$$

After membrane depolarization, the $I$ states absorb channels so that as the duration of the depolarization conditioning pulse becomes longer, the distribution of the channels shifts further and further to the right. After hyperpolarization (recovery phase), $A$ is an absorbing state, and the effective recovery rate becomes dependent on the distribution of channels between the different $I$ states. A similar one-dimensional diffusion model was theoretically explored by Millhauser et al. (1988) and was shown to result in power law distributions of the form demonstrated in the present study.

The results of the present report suggest that inactivation is a complex process that involves a mixture of time-independent and time-dependent rates. Moreover, Figure 7 shows that the relative amplitudes of the rates are time-dependent, an observation that was recently confirmed by Hayward et al. (1997) in a mammalian expression system. The fact that there are at least three distinct components of recovery from inactivation suggests that inactivation is composed of at least three distinct "families" of conformational states. One of these components (the rapid component) is time-independent, suggesting a simple internal structure (e.g., ball-and-chain-like mechanism) that is mechanistically unrelated

Figure 6. Scaling relationship between the conditioning duration and recovery time course that persists over a range of recovery voltages. $A$, Exemplar recovery time courses at three recovery voltages $(-60,-90$, and $-120 \mathrm{mV}$ ) are shown. The membrane is stepped to a $-10 \mathrm{mV}$ conditioning voltage for a duration $t(10,100$, or $300 \mathrm{sec})$. Recovery was measured by applying a series of test pulses to $-10 \mathrm{mV}$ and was normalized and plotted as described in Materials and Methods. Note that at all three recovery voltages, the time course of recovery becomes slower as the duration of the conditioning pulse is increased. At $-60 \mathrm{mV}$ compared with the other two voltages, the recovery time course seems to be more sensitive to the duration of the conditioning pulse. $B$, This becomes evident in this figure, where the value of the slower time constant $(\tau)$ is plotted as a function of the conditioning duration $t$. The slower time constants were extracted from the time course of recovery as shown in $A$. Filled circles are the mean values at $-120 \mathrm{mV}$; open circles are the mean values at $-60 \mathrm{mV}$. The scaling powers are $0.6(R>0.97)$ and $0.4(R>$ 0.99 ) for -60 and $-120 \mathrm{mV}$, respectively. 

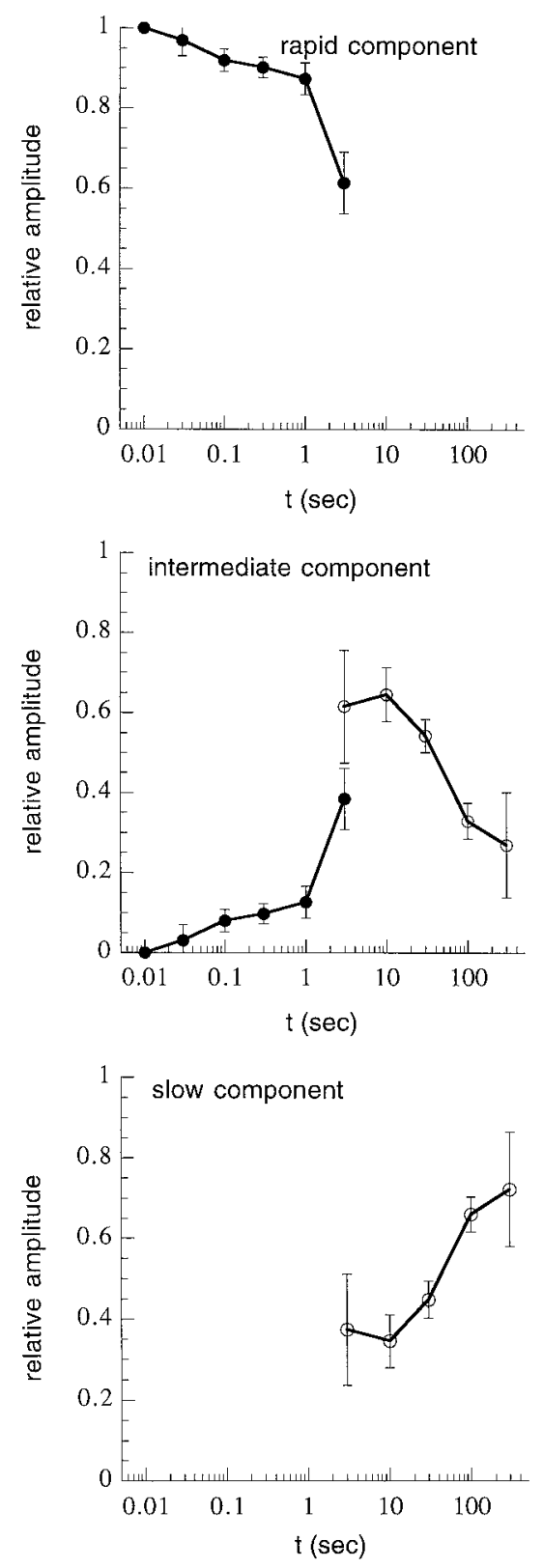
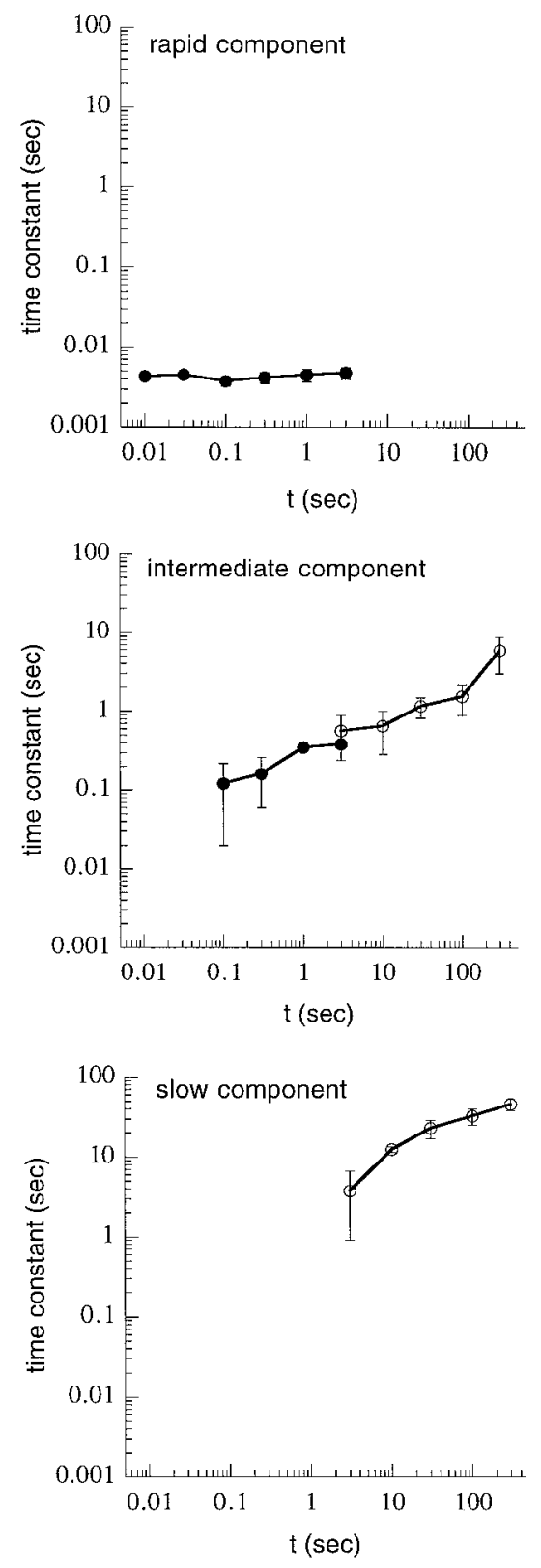

Figure 7. The relationships between different components of recovery from inactivation. The relative amplitudes and time constants of the rapid (top), intermediate (middle), and slow (bottom) recovery components are shown. Filled circles depict results of a double-pulse procedure; open circles depict results of a pulse-series procedure (see Pulse protocols and analysis of slow recovery in voltage-gated sodium channels in Materials and Methods). During long conditioning pulses $(>3 \mathrm{sec})$, the oocytes were kept under continuous perfusion at a rate of $\sim 2-4 \mathrm{ml} / \mathrm{min}$, a critical factor when a kinetic analysis of the intermediate component of recovery is sought (see Electrophysiological measurements in Materials and Methods). Note the dynamics of the relative amplitudes as the conditioning duration $t$ is increased. Also note that unlike that of the intermediate and slow components, the time constant of the rapid component is insensitive to the duration of the conditioning pulse. to slow inactivation. This conclusion conforms with more direct approaches to the problem of interaction between fast and slow inactivation in voltage-gated sodium channels (e.g., Valenzuela and Bennett, 1994; Featherstone et al., 1996). The other two components (the intermediate and the slow components) correspond to complex Markovian or fractal (non-Markovian) arrangements. The observation that the relative amplitudes of the three components change with time in an hierarchical manner (Fig. 7, left column; Hayward et al., 1997) simply reflects the fact that the three families of inactivation states are connected to each other in some manner.

\section{Neurophysiological implications}

Regardless of the mechanistic interpretation above, from a physiological point of view the significant observation is that the macroscopic availability of voltage-gated sodium channels is related in a scale-independent manner to the duration of previous activity. The effective time constant for moving between the two functionally defined states, $I \Rightarrow A$, is proportional to $t^{D}$, where $t$ is the time spent in the unavailable state of origin, and $D$ is a scaling power. Because $D$ is reported here to be positive, the longer the channel molecule resides at the state of origin (i.e., the inactivated pool), the harder it becomes to leave that state. This is a basis for scale-independent memory, as opposed to the scaledependent memory phenomenon that is familiar from standard descriptions of inactivation (e.g., fast inactivation and a refractory period that takes place within a uniquely defined milliseconds range). This scaling mechanism preserves traces of previous activity, over a wide range of time scales, in the form of modulated reaction rates rather than quantities of molecules that are available for activation [Marom (1998), his references]. In the case of the availability of voltage-gated sodium channels, one might expect such a scaling relationship to have implications on the firing patterns of neurons, because voltage-gated sodium 
$A$

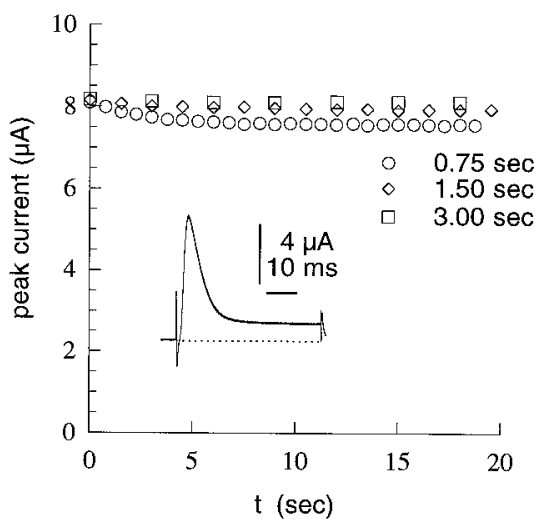

$B$

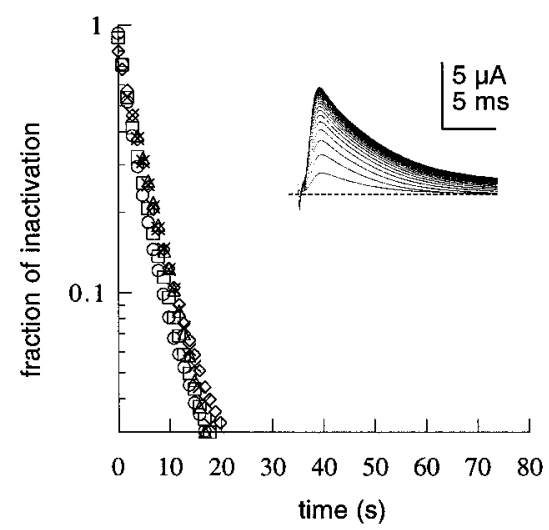

$C$

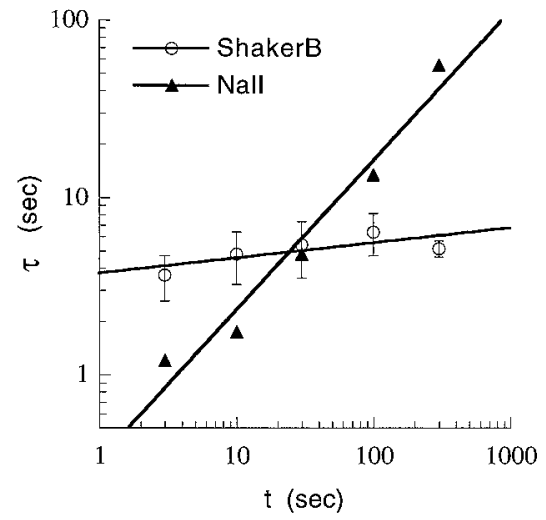

Figure 8. ShakerB channels recover from slow inactivation in a uniquely defined time scale. $A$, Evaluation of the duration of interpulse interval (at -90 $\mathrm{mV}$ ) that is required for avoidance of accumulation of inactivation because of 50-msec-long depolarizing test pulses to $+40 \mathrm{mV}$ is shown. These pulse parameters (amplitude and duration) allow for a completion of the N-type inactivation process. Slight accumulation of inactivation (saturating at $\cong 5 \%$ ) appears when pulses are delivered once every $750 \mathrm{msec}$ (open circles). Intervals of $1.5 \mathrm{sec}$ (open diamonds) and $3 \mathrm{sec}$ (open squares) allow for complete recovery between test pulses. Inset, Fourteen superposed current responses to identical 50-msec-long depolarizing voltage pulses from -90 to $+40 \mathrm{mV}$ separated by $1.5 \mathrm{sec}$ intervals at $-90 \mathrm{mV}$ are shown. $B$, ShakerB channels demonstrate a uniquely defined time scale for recovery from slow (C-type) inactivation. Slow inactivation was induced by conditioning depolarizations to $0 \mathrm{mV}$ from a holding potential of $-90 \mathrm{mV}$. The recovery interval, at -90 $\mathrm{mV}$, from the end of the conditioning pulse to the beginning of the series of test pulses lasted 1 sec. The fraction of inactivation was normalized and plotted as described in Materials and Methods, taking into account the positive sign of the currents. This time scale $(\cong 5$ sec) is independent of the duration $t$ of the conditioning depolarization (open circles, $t=3 \mathrm{sec}$; open squares, $t=10 \mathrm{sec}$; open diamonds, $t=30 \mathrm{sec} ; X, t=100 \mathrm{sec}$; and plus signs, $t=300 \mathrm{sec}$ ). Inset, Recovery from a $100 \mathrm{sec}$ conditioning depolarization to $0 \mathrm{mV}$ is shown. Recovery from slow inactivation is seen as a gradual increase in peak current responses to 20 -msec-long test pulses from -90 to $0 \mathrm{mV}$, which were delivered once per second. $C$, Comparison of the relationship between the duration of depolarization and the rate of recovery from slow inactivation of NaII ( filled triangles, mean values from Fig. $2 B$ ) and ShakerB (open circles, $n>3 ; \pm \mathrm{SD}$ ) is shown. Solid lines are power law functions of the form $\tau(t)=p \cdot t^{D}$ that were fit to the mean values (NaII, $p=0.3$; $D=$ $0.84 ; R=0.99$; ShakerB, $p=3.7 ; D=0.09 ; R>0.99)$.

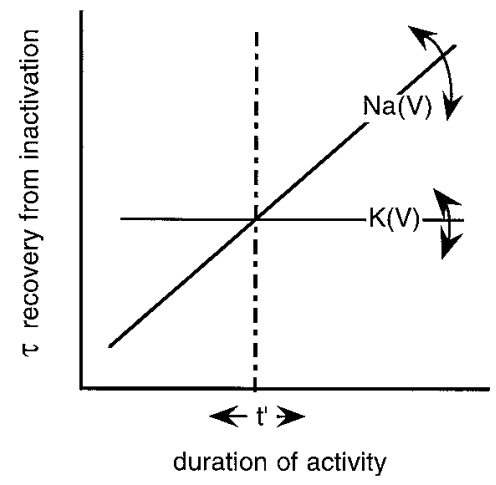

Figure 9. The nature of interaction between slow inactivation of voltagegated sodium $[N a(V)]$ and potassium $[K(V)]$ channels: an inference. A scheme of behavior for voltage-gated sodium and potassium channels. The position of the point of intersection (dashed line) and the relative slopes of the functions are variables that reflect the composition of ion channels that is unique to a neuron at a given point of time. It is suggested that these variables are dynamic (two-headed arrows) and influenced by the developmental stage and by activity.

channels can participate in a wide range of activity-dependent modulations over a wide range of time scales.

The importance of the channel behavior described here to neuron firing can be appreciated from Figure 9 (a schematic version of Fig. $8 C$ ). Note that the relationships between the rates of recovery from inactivation of sodium and potassium channels are such that during ongoing activity that lasts up to the point of intersection (depicted as $t^{\prime}$ ) each activation is followed by a net increase in excitability (recovery of the "restoring" potassium conductance from inactivation is slower than is recovery of the "exciting" sodium conductance). Beyond this point, each activa- tion results in suppression of activity (recovery of the exciting sodium conductance from inactivation is slower than is recovery of the restoring potassium conductance). Simplified as it is, the above picture suggests that the kinetic setpoints and the slopes of the $\tau(t)$ functions are important variables that are expected to determine in a nontrivial manner the dynamics of the neuronal response.

In summary, the results of this study suggest that neurons have a memory capacity that is embedded in the machinery of excitability and that is not delimited by particular time scales. Whether or not neurons use this capability is a subject for further research, but until this question is answered experimentally, there is no justification to assume locality in time when theorizing about the dynamics of threshold and firing patterns of single neurons and neurons in networks.

\section{REFERENCES}

Adelman WJ, Palti Y (1969) The effects of external potassium and long duration voltage conditioning on the amplitude of sodium currents in the giant axon of the squid, Loligo peali. J Gen Physiol 54:589-606.

Almers W, Stanfield PR, Stühmer W (1983) Slow changes in currents through sodium channels in frog muscle membrane. J Physiol (Lond) 339:253-271.

Auld VJ, Goldin AL, Krafte DS, Marshall J, Dunn JM, Catterall WA, Lester HA, Davidson N, Dunn RJ (1988) A rat brain $\mathrm{Na}^{+}$channel alpha subunit with novel gating properties. Neuron 1:449-461.

Bassingthwaighte JB, Liebovitch LS, West BJ (1994) Fractal physiology. New York: Oxford UP.

Brismar T (1977) Slow mechanism for sodium permeability inactivation in myelinated nerve fibre of Xenopus laevis. J Physiol (Lond) 270:283-297.

Catterall WA (1992) Cellular and molecular biology of voltage-gated sodium channels. Physiol Rev 72:S15-S48.

Chandler WK, Meves H (1970) Slow changes in membrane permeability and long lasting action potentials in axons perfused with fluoride solutions. J Physiol (Lond) 211:707-728. 
Cummins TR, Sigworth FJ (1996) Impaired slow inactivation in mutant sodium channels. Biophys J 71:227-236.

Featherstone DE, Richmond JE, Ruben PC (1996) Interaction between fast and slow inactivation in Skm1 sodium channels. Biophys J 71:3098-3109.

Fleidervish IA, Friedman A, Gutnick MJ (1996) Slow inactivation of $\mathrm{Na}^{+}$current and slow cumulative spike adaptation in mouse and guinea-pig neocortical neurons in slices. J Physiol (Lond) 493:83-97.

Fox JM (1976) Ultra-slow inactivation of the ionic currents through the membrane of myelinated nerve. Biochim Biophys Acta 426:232-244.

Hamill OP, Marty A, Neher E, Sakmann B, Sigworth FJ (1981) Improved patch-clamp techniques for high-resolution current recording from cells and cell-free membrane patches. Pflügers Arch 391:85-100.

Hayward LJ, Brown Jr RH, Cannon SC (1997) Slow inactivation differs among mutant $\mathrm{Na}$ channels associated with myotonia and periodic paralysis. Biophys J 72:1204-1219.

Hodgkin AL, Huxley AF (1952) A quantitative description of membrane current and its application to conduction and excitation in nerve. J Physiol (Lond) 117:500-544.

Hoshi T, Zagotta WN, Aldrich RW (1991) Two types of inactivation in Shaker $\mathrm{K}^{+}$channels: effect of alterations in the carboxy-terminal region. Neuron 7:547-556.

Isom LL, De Jongh KS, Catterall WA (1994) Auxiliary subunits of voltage-gated ion channels. Neuron 12:1183-1194.

Liebovitch LS (1989) Testing fractal and Markov models of ion channel kinetics. Biophys J 55:373-377.

Liebovitch LS, Sullivan JM (1987) Fractal analysis of a voltage-dependent potassium channel from cultured mouse hippocampal neurons. Biophys J 52:979-988.

Marom S (1998) Slow changes in the availability of voltage-gated ion channels: effects on the dynamics of excitable membranes [topical review]. J Membr Biol 161:107-116.
Millhauser GL, Salpeter EE, Oswald RE (1988) Diffusion models of ion-channel gating and the origin of power-law distributions from single-channel recording. Proc Natl Acad Sci USA 85:1503-1507.

Noda M, Ikeda T, Suzuki H, Takeshima H, Takahashi T, Kuno M, Numa S (1986) Expression of functional sodium channels from cloned cDNA. Nature 322:826-828.

Ruben PC, Starkus JG, Rayner MD (1992) Steady-state availability of sodium channels: interactions between activation and slow inactivation. Biophys J 61:941-955.

Ruben PC, Fleig A, Featherstone D, Starkus JP, Rayner MD (1997) Effects of clamp rise-time on rat brain IIA sodium channels in Xenopus oocytes. J Neurosci Methods 73:113-122.

Rudy B (1978) Slow inactivation of the sodium conductance in squid giant axons. Pronase resistance. J Physiol (Lond) 283:1-21.

Sauve R, Szabo G (1985) Interpretation of $1 / \mathrm{F}$ fluctuations in ion conducting membranes. J Theor Biol 113:501-516.

Schauf CL, Peneck TL, Davis FA (1976) Slow inactivation in Myxicola axons. Biophys J 16:771-778.

Simoncini L, Stühmer W (1987) Slow sodium channel inactivation in rat fast-twitch muscle. J Physiol (Lond) 383:327-337.

Stühmer W, Methfessel C, Sakmann B, Noda M, Numa S (1987) Patch clamp characterization of sodium channels expressed from rat brain cDNA. Eur Biophys J 14:131-138.

Tempel BL, Papazian DM, Schwarz TL, Jan YN, Jan LY (1987) Sequence of a probable potassium channel component encoded at Shaker locus of Drosophila. Science 237:770-775.

Valenzuela C, Bennett Jr PB (1994) Gating of cardiac $\mathrm{Na}^{+}$channels in excised membrane patches after modification by $\alpha$-chymotrypsin. Biophys J 67:161-171.

Wallner M, Weigle I, Meera P, Lotan I (1993) Modulation of the skeletal muscle sodium channel alpha subunit by the beta1 subunit. FEBS Lett 336:535-539. 